\section{Are aerobic interval training and continuous training isocaloric in coronary artery disease patients?}

\author{
Nele Pattyn', Véronique A Cornelissen', Roselien Buys', \\ Ann-Sofie Lagae', Jessica Leliaert' and Luc Vanhees ${ }^{1,2}$
}

European Journal of Preventive Cardiology $0(00) 1-10$

(C) The European Society of Cardiology 2016

Reprints and permissions: sagepub.co.uk/journalsPermissions.nav DOI: 10.1 I77/2047487316645468 ejpc.sagepub.com

@SAGE

\begin{abstract}
Background: Aerobic interval training (IT) seems to be superior to continuous training (CT) in improving exercise capacity (peak oxygen uptake $\left(\mathrm{VO}_{2}\right)$ ) in coronary artery disease $(\mathrm{CAD})$ patients in some, but not in all studies. Based on theoretical calculations, these comparative studies stated that the energy expenditure (EE) of both programmes is similar. To date, the caloricity of both programmes has never been objectively measured. Therefore, our aim was to objectively measure the EE of the IT and CT programmes described in the protocol of the SAINTEX-CAD study (based on Wisloff et al.; ITw and CTw), and the actually performed training intensities in the SAINTEX-CAD study by Conraads et al. (ITc and CTc).

Methods: Following a two-week run-in period with three IT and three CT training sessions, I8 male CAD patients (mean age $62.4 \pm 6.1$ years) performed four training sessions in random order on the cycle ergometer: an ITw, CTw, ITc and CTc test session. The EE was assessed by indirect calorimetry using gas exchange measurements obtained with the Oxycon mobile.

Results: We found a higher EE for CTc compared to ITc ( $352 \pm 90.8 \mathrm{kcal}$ versus $269 \pm 70.7 \mathrm{kcal} ; \mathrm{p}=0.026)$, while CTw and ITw seemed to be isocaloric $(317 \pm 85.2 \mathrm{kcal}$ versus $273 \pm 65.3 \mathrm{kcal} ; \mathrm{p}=0.42)$. Higher lactate levels were reached after IT sessions (ITw $5.42 \pm 1.42 \mathrm{mmol} / \mathrm{l}$, ITc $5.05 \pm 1.38 \mathrm{mmol} / \mathrm{l}$ ) compared to CT sessions (CTw $2.45 \pm 1.04 \mathrm{mmol} / \mathrm{l}$, CTc $3.4 \mathrm{I} \pm \mathrm{I} .44 \mathrm{mmol} / \mathrm{l})(\mathrm{p}<0.0 \mathrm{I})$. Lactate levels increased above baseline levels $(I .9 \mathrm{I} \pm 0.34 \mathrm{mmol} / \mathrm{l})$ except for the CTw session.

Conclusion: CTc expended significantly more energy compared to ITc, showing that the programmes used in the SAINTEX-CAD study were not isocaloric. In contrast, isocaloricity was met for CTw and ITw.
\end{abstract}

\title{
Keywords
}

Isocaloric, energy expenditure, indirect calorimetry, Oxycon mobile, interval training, aerobic exercise, coronary artery disease

Received I5 January 2016; accepted 31 March 2016

\section{Introduction}

Coronary artery disease (CAD) causes about $45 \%$ of all deaths in Europe. ${ }^{1}$ Exercise-based cardiac rehabilitation is an effective intervention in the prevention and management of CAD. ${ }^{2}$ Substantial evidence shows increases in maximal exercise capacity (peak oxygen uptake $\left(\mathrm{VO}_{2}\right)$ ) after cardiac rehabilitation, ${ }^{3}$ with associated decreases in cardiac morbidity and mortality. ${ }^{4}$ Therefore, the main aim of exercisebased cardiac rehabilitation is to improve peak $\mathrm{VO}_{2}$. Its effectiveness, however, depends on the intensity, duration, frequency and type of exercise. ${ }^{2}$ The search for an optimal training programme is still ongoing and results of comparative studies remain controversial. $^{5}$

The most common training programmes currently used in cardiac rehabilitation are continuous training (CT) and interval training (IT) or a mixture. CT is

\footnotetext{
'Department of Rehabilitation Sciences, KU Leuven, Belgium

${ }^{2}$ Department of Cardiovascular Diseases, University Hospitals of Leuven, Belgium

\section{Corresponding author:}

Luc Vanhees, Department of Rehabilitation Sciences, Tervuursevest I0I, B I50I B 300I, Leuven, Belgium.

Email: luc.vanhees@kuleuven.be
} 
usually performed at an intensity of $40-80 \%$ of the peak $\mathrm{VO}_{2}{ }^{2}$ A decade ago, it was shown that IT, consisting of several bouts of exercise at a higher intensity $\left(80-90 \%\right.$ of the peak $\left.\mathrm{VO}_{2}\right)$ alternated by active recovery periods, may provide a safe alternative to $\mathrm{CT}$ in $\mathrm{CAD}$ patients. ${ }^{6}$ However, the results from subsequent comparative studies are conflicting and inconsistent with some studies showing that IT and CT equally improve peak $\mathrm{VO}_{2}{ }^{7-10}$ while others suggest that IT is superior to CT. ${ }^{6,11-14}$ One of the possible reasons for these inconsistencies in literature might be that the training protocols not only differ in intensity, but also in energy expenditure (EE). Indeed, at the population level significant correlations are found between peak $\mathrm{VO}_{2}$ and physical activity EE and also between changes in these variables. ${ }^{15}$ Furthermore, Church et al. demonstrated a graded dose-response change in physical fitness with increasing EE of the exercise training programmes at a constant exercise intensity. ${ }^{16}$ In line with this, Vanhees et al. showed that exercise intensity and frequency are independent determinants of the change in peak $\mathrm{VO}_{2} \cdot{ }^{17}$ Given that the duration of the training sessions was similar, a higher exercise frequency will result in a higher exercise $\mathrm{EE}$, leading to higher increases in peak $\mathrm{VO}_{2} \cdot{ }^{17}$

Three recent meta-analyses documented that IT results in a significantly larger effect on peak $\mathrm{VO}_{2}$ when compared to $\mathrm{CT}$ in CAD patients. ${ }^{18-20}$ The effect of IT on peak $\mathrm{VO}_{2}$ was shown to be almost twice the amount of the effect of CT $(20.5 \%$ vs $12.8 \%) .{ }^{19}$ Of the nine studies included in the meta-analysis of Pattyn et al., ${ }^{19}$ seven reported to compare isocaloric training programmes ${ }^{6,8,11,21-24}$ and two did not mention EE. ${ }^{7,9}$ For example, in the first comparative IT versus CT study in CAD patients, Rognmo et al. compared training programmes with the same total training workload calculated by the total $\mathrm{VO}_{2}$-time relationship. The calculated average peak $\mathrm{VO}_{2}$ of all subjects during the two exercise intensities of the IT protocol $(3 \times 3 \mathrm{~min}$ at $60 \%$ of peak $\mathrm{VO}_{2}$ and $4 \times 4 \mathrm{~min}$ at $90 \%$ of peak $\mathrm{VO}_{2}$ ) was used to derive the duration of the $\mathrm{CT}$ protocol $\left(60 \%\right.$ of peak $\left.\mathrm{VO}_{2}\right)$ required to yield the same workload. ${ }^{6}$ Later studies referred to this method for designing isocaloric training programmes. ${ }^{11,22,25}$ Two other methods for calculating training workload, based on heart rate (HR), include Banister's training impulse method (the TRIMP method), used by Iellamo et al., ${ }^{23}$ and Edwards' HR-based method. ${ }^{26}$

To the best of our knowledge, however, no study objectively measured the actual EE of IT and CT. Moreover, some studies did not describe or refer to the formulae used to document the isocaloricity of their training programmes. ${ }^{8,24}$

The purpose of our study is therefore to objectively measure the actual EE of the IT and CT programmes as described in the protocol of the SAINTEX-CAD study (based on Wisloff et al.), ${ }^{22}$ and the actually performed training intensities of the SAINTEX-CAD study. ${ }^{10}$

\section{Methods}

\section{Participants}

Our study included 20 male CAD patients (mean age $62.4 \pm 6.1$ ), referred to the Cardiac Rehabilitation Unit from the University Hospitals of Leuven between July 2014-November 2015. Subjects signed a written informed consent prior to participation in accordance with the Declaration of Helsinki. ${ }^{27}$ The study was approved by the local ethics committee (Commissie Medische Ethiek KU Leuven).

The inclusion criteria were: (a) patients with CAD without heart failure, acute myocardial infarction (AMI), percutaneous coronary intervention (PCI) and/or coronary artery bypass grafting (CABG); (b) left ventricular ejection fraction (LVEF) higher than $40 \%$; (c) under optimal medical care; (d) stable with regard to symptoms and pharmacotherapy for at least four weeks; and (e) having completed at least three conventional cardiac rehabilitation training sessions, with a maximum of 15 .

A flowchart of the trial is shown in Figure 1. After inclusion, two patients dropped out before any tests were performed: one patient because of an old knee injury and one due to severe arrhythmias at high training intensities. There were no significant differences between the baseline characteristics of the patients with or without the dropouts (data not shown).

\section{Measurements}

Anthropometric measurements. Height $(\mathrm{cm})$ and weight $(\mathrm{kg})$ were measured, and body mass index (BMI) $\left(\mathrm{kg} / \mathrm{m}^{2}\right)$ was calculated by dividing weight $(\mathrm{kg})$ by height squared $\left(\mathrm{m}^{2}\right)$.

Cardiopulmonary exercise test. Before starting the cardiac rehabilitation, subjects performed a maximal graded exercise test on a cycle ergometer (Oxycon Pro, Jaeger, CareFusion, Germany) supervised by a trained exercise physiologist. A protocol of 20 watts (W) $+20 \mathrm{~W} / \mathrm{min}$ until exhaustion was used. During the test, breath-by-breath gas exchange measurements were monitored continuously, allowing online determination of ventilation (VE), oxygen uptake $\left(\mathrm{VO}_{2}\right)$, and carbon dioxide production $\left(\mathrm{VCO}_{2}\right)$. In addition, a 12-lead electrocardiogram was continuously registered. The peak HR was defined as the highest HR reached at the end of the test. The peak $\mathrm{VO}_{2}$ was determined as the 


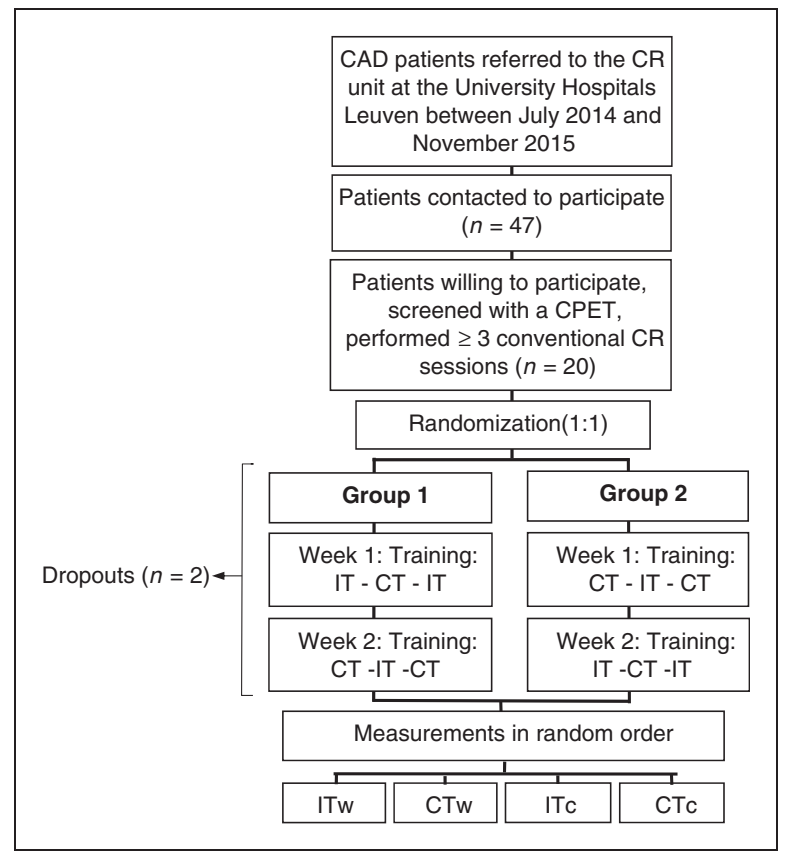

Figure I. Flowchart of the trial. CAD: coronary artery disease; CPET: cardiopulmonary exercise test; CR: cardiac rehabilitation; $\mathrm{CT}$ : continuous training; CTc: continuous training according to the SAINTEX-CAD study of Conraads et al.; ${ }^{10} \mathrm{CTw}$ : continuous training according to the study of Wisloff et al.;2 IT: interval training; ITc: interval training according to the SAINTEX-CAD study of Conraads et al.; ${ }^{10}$ ITw: interval training according to the study of Wisloff et al. ${ }^{22}$

$\mathrm{VO}_{2}$ during the last full bout of $30 \mathrm{~s}$ of the test. A peak respiratory exchange ratio of at least 1.10 defined a maximal effort. ${ }^{28}$ Individual peak $\mathrm{VO}_{2}$ results were compared with predicted reference values of Wasserman et al. to determine percentage of predicted peak $\mathrm{VO}_{2}{ }^{29}$ The first ventilatory threshold was defined as 'the nadir or first increase of ventilation (VE) over oxygen uptake $\left(\mathrm{O}_{2}\right)\left(\mathrm{VE} / \mathrm{VO}_{2}\right)$ versus workload without a simultaneous increase in VE over carbon dioxide production $\left(\mathrm{VCO}_{2}\right)\left(\mathrm{VE} / \mathrm{VCO}_{2}\right)$ versus workload' (p 730, Table 2). ${ }^{30}$ The second ventilatory threshold was defined as 'the nadir or non-linear increase of $\mathrm{VE} /$ $\mathrm{VCO}_{2}$ versus workload'. ${ }^{30}$

\section{IT and CT familiarisation sessions}

After inclusion, subjects were randomly assigned on a 1:1 base to either training group 1 (starting with IT) or group 2 (starting with CT). They performed a total of six supervised IT $(n=3)$ and CT $(n=3)$ sessions on a cycle ergometer (Ergo-fit, Gymna, Brussels, Belgium), three times per week for two weeks. The IT and CT sessions were alternated and aimed to familiarise the subjects with both training programmes. A Garmin chest strap and wristwatch continuously monitored HR (Garmin, Garmin International, Kansas, USA), and training loads were adapted throughout the training to ensure that participants would remain within the prescribed HR zones.

\section{IT and CT test sessions}

The two-week run-in period was followed by four test sessions within two weeks: the IT and CT according to the protocol of the SAINTEX-CAD study, ${ }^{25}$ which was based on Wisloff et al. (ITw and CTw), ${ }^{22}$ and the IT and $\mathrm{CT}$ according to the actually achieved intensities in the SAINTEX-CAD study of Conraads et al. (ITc and $\mathrm{CTc}) .{ }^{10}$ An online randomisation procedure was performed to determine the sequence of the four test sessions. A visual representation of the IT and CT programmes including their specific training intensities and durations is shown in Figure 2.

During the test sessions, HR was continuously monitored using a Polar chest strap (Polar, Polar Electro, Kempele, Finland). Training loads were adapted throughout the test to ensure that participants would remain within the prescribed HR zones. Each patient performed all four test sessions at the same time in the morning $(0800,0930$ or 1100$)$. As beta-blocking medication might influence HR, we also asked our patients to take their medication at the same time each morning of the test.

Breath by breath gas exchange measurements $\left(\mathrm{VE}, \mathrm{VO}_{2}, \mathrm{VCO}_{2}\right)$, averaged each $30 \mathrm{~s}$, were provided by the Oxycon mobile device (Oxycon mobile Jaeger, CareFusion, Germany) and were used to calculate the EE (indirect calorimetry) according to the American College of Sports Medicine (ACSM) guidelines; one litre of $\mathrm{O}_{2}$ uptake equals $5 \mathrm{kcal}$.

Lactate levels were determined at baseline; at the end of each four-minute interval for the IT sessions; and at 10, 20, 30 and $37 \mathrm{~min}$ of the continuous bout for the CT session, using the Lactate Pro 2 device (Lactate Pro 2, Arkray, Shiga, Japan).

\section{Statistical analysis}

All values are expressed as mean \pm standard deviation (SD), median and range, or as number and percentage. Statistical analyses were performed in SAS 9.3 (SAS Institute, Cary, North Carolina, USA). All data were normally distributed according to the Shapiro-Wilk test for normality. To examine the differences in EE, exercise intensity or lactate between the ITw, CTw, ITc and $\mathrm{CTc}$ session, a repeated measures analysis of variance (ANOVA) was performed. The Scheffé test for multiple comparisons was used as a post-hoc test. A $p$-value $<0.05$ was considered statistically significant. 


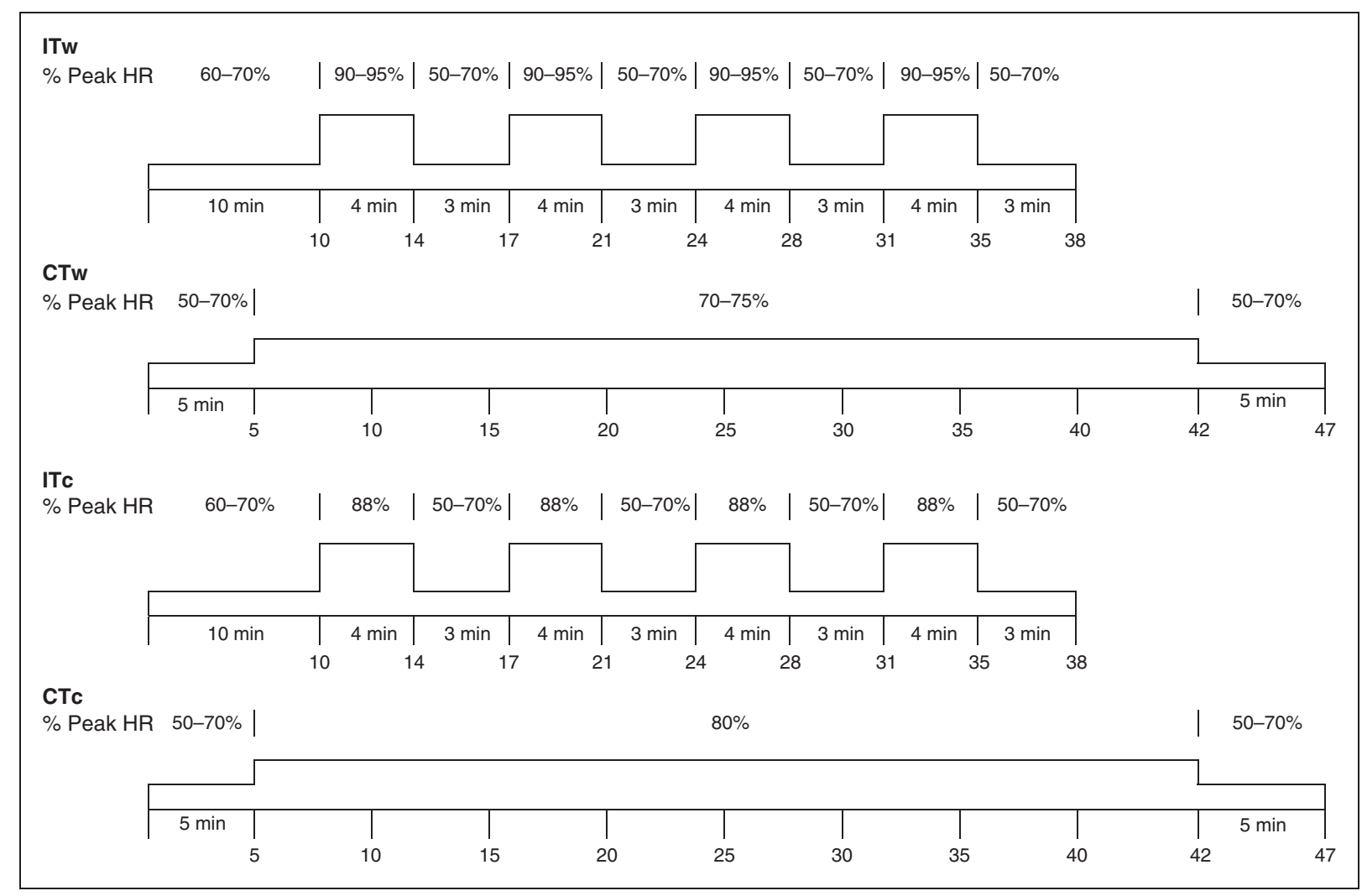

Figure 2. A visual presentation of the interval training (IT) and continuous training (CT) exercise programmes. CTc: continuous training according to the SAINTEX-CAD study of Conraads et al.; ${ }^{10} \mathrm{CTw}$ : continuous training according to the study of Wisloff et al.; ${ }^{22}$ ITc: interval training according to the SAINTEX-CAD study of Conraads et al.; ${ }^{10}$ ITw: interval training according to the study of Wisloff et al.; ${ }^{22} \%$ peak HR: percentage of peak heart rate.

As this is the first study to evaluate EE of different exercise programmes, without precedence on which to base accurate power calculations, a sample size calculation could not been performed. However, at completion of the study, we performed a post-hoc power calculation. For a repeated measures ANOVA (within factors) with an $\alpha$ probability of 0.05 , a total sample size of 18 and a calculated effect size of 0.45 we had a power of $99.6 \%$ to detect significant differences in EE between the four exercise sessions.

$\mathrm{HR}(\mathrm{bpm})$ and $\mathrm{VO}_{2}(\mathrm{ml} / \mathrm{min})$ were averaged every $30 \mathrm{~s}$ during the tests. To calculate the $\mathrm{EE}$ with the ACSM formula, data of the total session measured per $30 \mathrm{~s}$ (IT $38 \mathrm{~min}$; CT $47 \mathrm{~min}$ ) were used. In order to document the training intensities, $\mathrm{HR}$ and $\mathrm{VO}_{2}$ measurements were averaged for the total four-minute high intensity bouts $(4 \times 4 \mathrm{~min})$, the total three-minute recovery bouts $(4 \times 3 \mathrm{~min})$ and the total continuous bout ( $37 \mathrm{~min}$ ) (total $\mathrm{HR}$ and $\mathrm{VO}_{2}$ ), and expressed as a percentage of the peak $\mathrm{HR}$ and $\mathrm{VO}_{2}$. In addition, the $\mathrm{HR}$ and $\mathrm{VO}_{2}$ values of the last minute of each fourminute interval and the last minute of each three- minute recovery bout for IT, and at 10', 20', 30' and $37^{\prime}$ for CT were averaged, after which an additional average was calculated of these four measurements (end $\mathrm{HR}$ and $\mathrm{VO}_{2}$ ). These values were also expressed as a percentage of the peak $\mathrm{HR}$ and $\mathrm{VO}_{2}$.

\section{Results}

A total of 18 patients completed the six training sessions and the four tests; baseline characteristics are presented in Table 1. Patients did not change medication during the study period.

Figure 3 shows a consistent pattern for total EE in all participants, with CTc expending the largest amount of energy, followed by CTw (except in participant 1 and 10) and then ITw and ITc. In Figure 4 and Table 2, the mean EE per training session is presented. We found that CTw and ITw were isocaloric $(p=0.42)$, while CTc expended significantly more energy compared to ITc $(p=0.026)$ (Table 2). Moreover, CTc resulted in a higher EE compared to ITw $(p=0.037)$. 


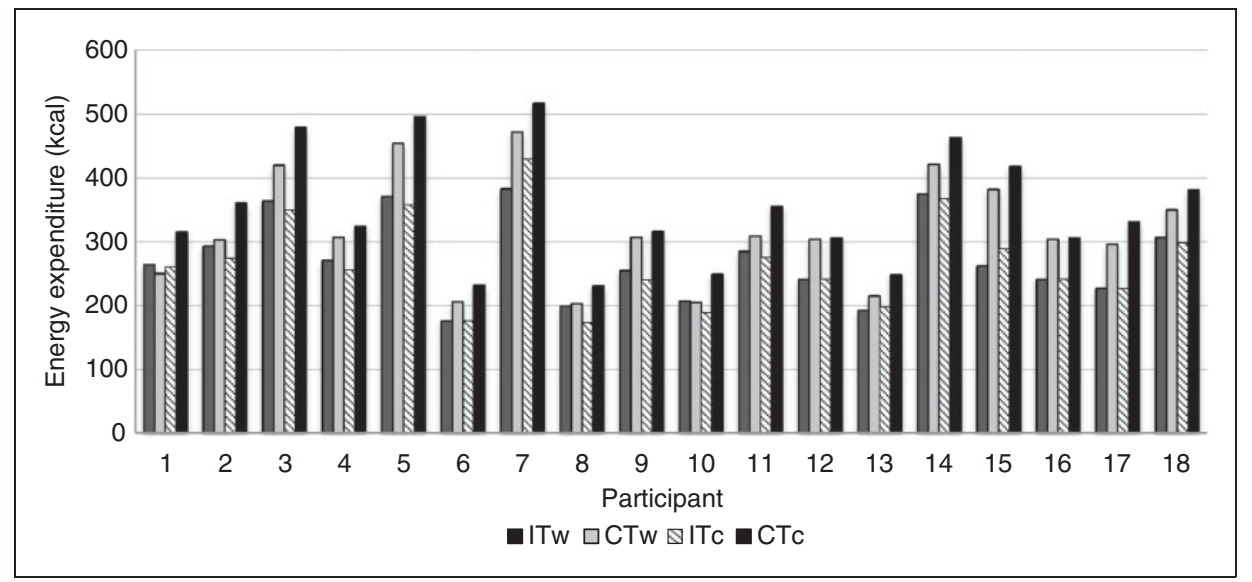

Figure 3. Energy expenditure per participant for each test. CTc: continuous training according to the SAINTEX-CAD study of Conraads et al.; ${ }^{10} \mathrm{CTw}$ : continuous training according to the study of Wisloff et al.; ${ }^{22}$ ITc: interval training according to the SAINTEX-CAD study of Conraads et al.; ${ }^{10}$ ITw: interval training according to the study of Wisloff et al. ${ }^{22}$

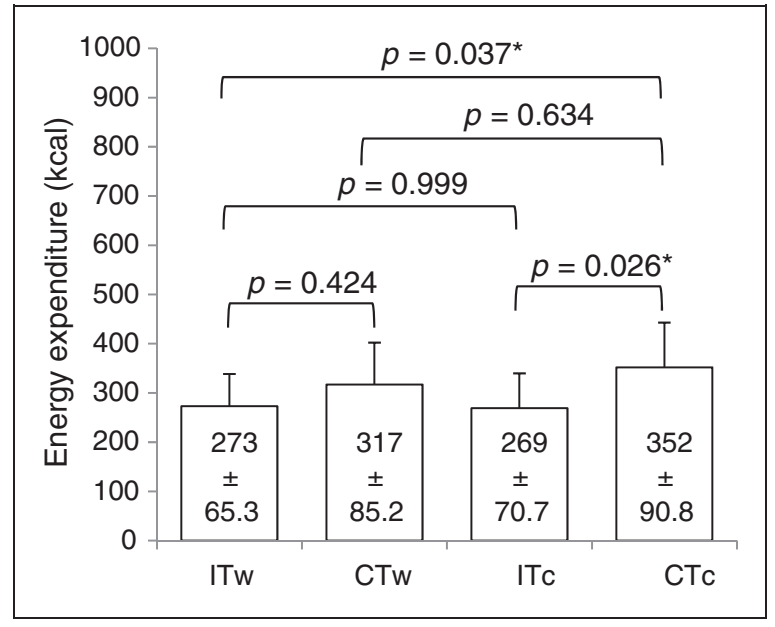

Figure 4. Comparison of the energy expenditures according to the study of Wisloff et al. ${ }^{22}$ and according to the SAINTEX-CAD study of Conraads et al. ${ }^{10}$ Other not-mentioned comparisons were not significantly different. CTc: continuous training according to the SAINTEX-CAD study of Conraads et al.; ${ }^{10}$ $C T w$ : continuous training according to the study of Wisloff et al.; ${ }^{22}$ ITc: interval training according to the SAINTEX-CAD study of Conraads et al.; ${ }^{10}$ ITw: interval training according to the study of Wisloff et al.,22* $\mathrm{p}<0.05$.

after CTw, compared to resting lactate levels; and (d) significantly higher lactate levels after IT sessions compared to CT sessions.

Rognmo et al. ${ }^{6}$ used similar IT and CT protocols as Wisloff et al. ${ }^{22}$ in CAD patients and found that IT was superior to $\mathrm{CT}$ in improving peak $\mathrm{VO}_{2}(+17.9 \%$ versus $+7.9 \%$ ); Conraads et al. reported that both protocols were equally effective in improving peak $\mathrm{VO}_{2}$ $(+22.7 \%$ versus $+20.3 \%){ }^{10}$ However, Conraads et al. came to the conclusion that a number of CAD patients were incapable of maintaining $90-95 \%$ of their peak HR throughout the four-minute interval, and they moreover observed that the intensity of $\mathrm{CT}$ in the prescribed protocol $\left(70-75 \%\right.$ of peak HR) ${ }^{6,22}$ was probably insufficient to achieve usual improvements ${ }^{3}$ in peak $\mathrm{VO}_{2}$. These different training intensities (ITc lower and CTc higher than prescribed) may explain the differences in results and conclusions between these studies. ${ }^{10}$ In addition, Conraads et al. consequently advised that $\mathrm{CT}$ should be set at an intensity higher than $70-75 \%$ of peak HR, which can still be sustained for $37 \mathrm{~min}^{10}$

In the current study, all subjects (except two) were able to perform the ITw test within the predefined HR zones, with an average of $91.2 \pm 3.7 \%$ peak HR at the end of the high intensity interval. Nevertheless, most of the patients had difficulties maintaining this high HR zone and needed constant encouragement from the supervising exercise physiologist. The intensity set at $93 \%$ of peak HR was above the second ventilatory threshold $(88.6 \%$ of peak HR, Table 1$)$, which makes this effort very difficult to sustain. During the ITc session, intensity was set at $88 \%$ of peak HR, just below the second ventilatory threshold, which was clearly easier to tolerate. Despite the differences in training HR, EE during ITw and ITc seemed to be similar (ITw $273 \mathrm{kcal}$ vs ITc $269 \mathrm{kcal}$ ). Nevertheless, Moholdt et al. showed that even within the high intensity training zones, exercise intensity was an important determinant for improving peak $\mathrm{VO}_{2}$, with categories $<88 \%$ and $>92 \%$ of peak HR resulting in significantly different increases. ${ }^{32}$ Based on this information, it seems that training intensity is a more important determinant for achieving favourable training responses compared to $\mathrm{EE}$ (training volume $=$ duration $\times$ intensity), 
Table 2. Energy expenditure, lactate levels and training characteristics for each test.

\begin{tabular}{|c|c|c|c|c|c|c|}
\hline Variable & ITw & CTw & ITc & CTc & $p$-Value & Post-hoc Scheffé results \\
\hline Energy expenditure (kcal) & $273 \pm 65.3$ & $317 \pm 85.2$ & $269 \pm 70.7$ & $352 \pm 90.8$ & $p=0.006$ & $\mathrm{ITw}<\mathrm{CT}$ c; ITc $<$ CTc \\
\hline Mean lactate $(\mathrm{mmol} / \mathrm{l})$ & $5.42 \pm 1.42$ & $2.45 \pm 1.04$ & $5.05 \pm 1.38$ & $3.4 \mathrm{I} \pm \mathrm{I} .44$ & $p<0.001$ & $\begin{array}{l}\text { ITw }>\text { CTw; ITc }>\text { CTc; } \\
\quad \text { ITw }>\text { CTc; ITc }>\text { CTw }\end{array}$ \\
\hline \multicolumn{7}{|l|}{ Training intensity } \\
\hline \% Peak HR prescribed (interval) & $90-95$ & $70-75$ & 88 & 80 & & \\
\hline \% Peak HR end interval & $91.2 \pm 3.7$ & $73.6 \pm 1.1$ & $87.1 \pm 1.9$ & $79.7 \pm 1.6$ & $p<0.001$ & $\begin{array}{l}\text { ITw }>\text { CTw; ITc }>\text { CTc; } \\
\text { ITw }>\text { CTc; ITc }>\text { CTw; } \\
\text { ITw }>\text { ITc; CTw }>\text { CTc }\end{array}$ \\
\hline \% Peak HR total interval & $86.0 \pm 3.7$ & $73.1 \pm 0.9$ & $82.7 \pm 2.6$ & $78.3 \pm 1.9$ & $p<0.001$ & $\begin{array}{l}\text { ITw }>\text { CTw; ITc }>\text { CTc; } \\
\text { ITw }>\text { CTc; ITc }>\text { CTw; } \\
\text { ITw }>\text { ITc; CTw }>\text { CTc }\end{array}$ \\
\hline \% Peak HR prescribed (recovery) & $50-70$ & $50-70$ & $50-70$ & $50-70$ & & \\
\hline$\%$ Peak HR end recovery & $7 I .1 \pm 5.2$ & $65.2 \pm 4.5$ & $68.6 \pm 4.3$ & $69.2 \pm 4.5$ & $p=0.003$ & $\mathrm{ITw}>\mathrm{CTw}$ \\
\hline \% Peak HR total recovery & $75.7 \pm 4.9$ & $66.9 \pm 3.9$ & $72.9 \pm 3.5$ & $71.2 \pm 3.9$ & $p<0.001$ & $\begin{array}{l}\text { ITw }>\text { CTw; ITw }>\text { CTc; } \\
\quad I T c>C T w ; C T c>C T w\end{array}$ \\
\hline$\%$ Peak $\mathrm{VO}_{2}$ end interval & $104 \pm 13.4$ & $75.5 \pm 10.9$ & $100 \pm 11.5$ & $85.2 \pm 11.9$ & $p<0.001$ & $\begin{array}{l}\text { ITw }>\text { CTw; ITc }>\text { CTc; } \\
\quad I T w>C T c ; \text { ITc }>\text { CTw }\end{array}$ \\
\hline$\%$ Peak $\mathrm{VO}_{2}$ total interval & $92.2 \pm 11.0$ & $74.6 \pm 10.7$ & $89.9 \pm 10.0$ & $83.6 \pm 10.9$ & $p<0.001$ & $\mathrm{ITw}>\mathrm{CTw}$; ITc $>$ CTw \\
\hline$\%$ Peak $\mathrm{VO}_{2}$ end recovery & $58.9 \pm 8.8$ & $55.1 \pm 9.8$ & $58.4 \pm 7.4$ & $55.3 \pm 8.4$ & $p=0.40$ & \\
\hline$\%$ Peak $\mathrm{VO}_{2}$ total recovery & $71.9 \pm 10.3$ & $60.0 \pm 9.6$ & $70.4 \pm 8.4$ & $60.7 \pm 8.1$ & $p<0.001$ & $\begin{array}{l}\text { ITw > CTw; ITc > CTc; } \\
\quad I T w>C T c ; \text { ITc }>\text { CTw }\end{array}$ \\
\hline
\end{tabular}

CTc: continuous training according to the SAINTEX-CAD study of Conraads et al.: ${ }^{10} \mathrm{CTw}$ : continuous training according to the study of Wisloff et al.; ${ }^{22}$ HR: heart rate; ITc: interval training according to the SAINTEX-CAD study of Conraads et al.; ${ }^{10}$ ITw: interval training according to the study of Wisloff et al.; ${ }^{22} \mathrm{VO}_{2}$ : oxygen uptake.

as both IT sessions seem to be similar in volume and duration.

Whereas also the CT sessions seemed to be similar in EE (CTw $317 \mathrm{kcal}$ vs CTc $352 \mathrm{kcal}$ ), an 11\% difference ( $35 \mathrm{kcal})$ per session was found. As the durations were similar, the small but not significant differences in intensity might explain the larger gain in peak $\mathrm{VO}_{2}$ in the CT group of the SAINTEX-CAD study $( \pm 20.3 \%)^{10}$ compared to the study of Rognmo et al. $( \pm 7.9 \%){ }^{6}$

Combining the previous results of the SAINTEXCAD study (IT and CT equally improved peak $\mathrm{VO}_{2}$ ) with the present findings, we can conclude that IT is more efficient than CT because of a significantly lower energy cost and training duration for an equal improvement in peak $\mathrm{VO}_{2}$. This is in line with metaanalyses ${ }^{18-20}$ aggregating results of IT and CT on peak $\mathrm{VO}_{2}$ in CAD patients, revealing a superior effect following IT. However, isocaloricity and EE of training sessions is not a goal on its own. The most important question remains which training modality is the most appropriate to increase peak $\mathrm{VO}_{2}$, and it seems that both IT and CT are equally effective when sufficiently high intensities are achieved in the CT protocol.
Patients should be able to choose their preferred training modality in order to increase their intrinsic motivation for a lifelong physically active lifestyle.

On the other hand, EE is important for weight loss. Our findings support the overall results on body weight in the meta-analysis of Pattyn et al. and Liou et al. ${ }^{19,20}$ showing that CT is more effective in reducing body weight since more calories are expended compared to IT. Performing 36 sessions ( 12 weeks, $3 \times /$ week) of CT leads to a $3000 \mathrm{kcal}(31 \%)$ higher expenditure compared to IT, which equals a difference of almost $0.5 \mathrm{~kg}$ fat loss. Thus, we can conclude that IT is more efficient in improving peak $\mathrm{VO}_{2}$, while $\mathrm{CT}$ at sufficiently high intensities expends more energy leading to larger reductions in body weight.

According to Skinner's three-phase model, training modalities below the first ventilatory threshold (60$70 \%$ peak HR) do not exceed a $2 \mathrm{mmol} / 1$ lactate level. ${ }^{30,33}$ When lactate levels exceed $4 \mathrm{mmol} / 1$, passing the second ventilatory threshold, the exercise is considered anaerobic ( $>90 \%$ peak HR). In our study, average lactate levels during the training sessions were between $2.45 \mathrm{mmol} / 1$ (CTw) and $5.42 \mathrm{mmol} / 1$ (ITw). Statistical analysis revealed a significant difference 


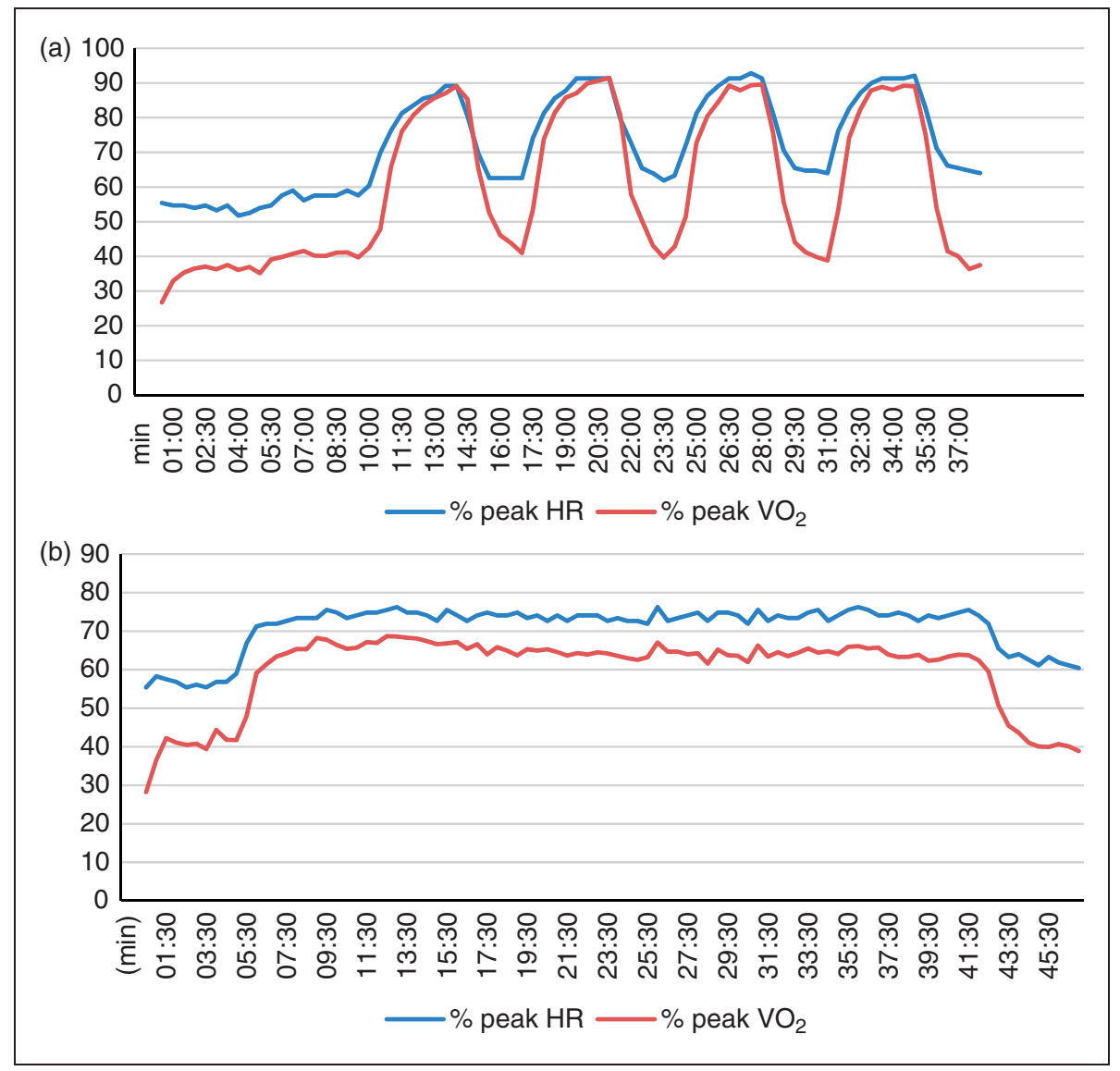

Figure 5. Example of the heart rates and oxygen uptakes during (a) initial training and (b) continuous training sessions in a patient under beta-blockade. CTw: continuous training according to the study of Wisloff et al.:22 HR: heart rate; ITw: interval training according to the study of Wisloff et al.; ${ }^{22} \mathrm{VO}_{2}$ : oxygen uptake.

between the lactate levels of the CT and IT, with IT showing lactate values $>4 \mathrm{mmol} / \mathrm{l}$. This implies that the high intensity intervals may have been performed anaerobically for most of the individuals (ITw 14/18; ITc 13/18), but not in all, since this secondary threshold is highly individual. The frequently used term 'aerobic' interval training may thus be inappropriate and misleading, but further research is needed. We can however conclude that the participants remained in the aerobic zone for both CT sessions (CTw $2.45 \mathrm{mmol} / 1$ and CTc $3.41 \mathrm{mmol} / \mathrm{l})$. From the lactate levels of the CTw session, we can conclude that an intensity set at $70-75 \%$ of peak HR is simply insufficient as a training stimulus.

\section{Limitations}

The results of this study should be interpreted within the context of its limitations. The first limitation of our study consists of a relatively small sample size. However, our results were highly consistent and the statistical power to detect significant differences in EE between the four sessions was $99.6 \%$.
Second, compared to the Oxycon Pro (a stationary apparatus, used for the maximal exercise test), the Oxycon Mobile (a portable device), which was used for the test sessions, might overestimate $\mathrm{VO}_{2}$ when the intensity exceeds $200 \mathrm{~W} .{ }^{34}$ Since only one participant went just beyond $200 \mathrm{~W}$ during his IT test session, we can consider that our measurements from the Oxycon Mobile were sufficiently accurate.

Third, we determined the HR zones based on one single maximal exercise test. However, many factors can influence this test, which may generate a deviation of the peak HR, resulting in an over or underestimation of the intensity. We expect, however, these variations to be random across all subjects, which should, therefore, not significantly influence our final result. ${ }^{32}$ However, this could have led to the fact that two patients were not able to achieve the prescribed intensity during the ITw session.

Fourth, patients already performed at least three conventional training sessions and six familiarisation sessions ( $>3$ weeks) before starting the tests. As we previously reported that substantial changes in peak $\mathrm{VO}_{2}$ 
and peak HR already occur after six weeks of training, ${ }^{10}$ this run-in period could have influenced the high $\mathrm{VO}_{2}$ levels achieved at prescribed HR. For example, patients trained at $100-104 \%$ of peak $\mathrm{VO}_{2}$ during IT, and $76-85 \%$ of peak $\mathrm{VO}_{2}$ during CT, which is physiologically impossible without a run-in period.

Fifth, we did not measure lactate levels during the maximal exercise test, hence we were unable to compare these individual maximal lactate levels to the lactate levels found during the tests.

\section{Conclusion}

We found that CT according to the SAINTEX-CAD study expended significantly more energy than IT. As previous studies showed IT and CT to either yield similar improvement in peak $\mathrm{VO}_{2}$, or a larger improvements after IT compared to CT, we can conclude that IT is more efficient in improving peak $\mathrm{VO}_{2}$, producing a larger gain during a shorter training duration and at a lower energy cost. However, since EE is not a goal on its own in cardiac rehabilitation, the main question remains which training modality is the most appropriate to increase peak $\mathrm{VO}_{2}$. We can suggest that IT and CT are equally effective, if $\mathrm{CT}$ sessions are performed at sufficiently high intensities.

We stress the importance of objectively measuring actual caloric expenditure by indirect calorimetry in pilot studies, rather than using a general formula for setting up isocaloric exercise training programmes.

\section{Acknowledgements}

All authors take responsibility for all aspects of the reliability and freedom from bias of the data presented and their discussed interpretation.

\section{Declaration of conflicting interests}

The authors declared no potential conflicts of interest with respect to the research, authorship, and/or publication of this article.

\section{Funding}

The authors disclosed receipt of the following financial support for the research, authorship, and/or publication of this article: VAC is funded as a postdoctoral fellow and received research funding from the Research Foundation Flanders (FWO). RB has been awarded research grants from the FWO and from the Belgian Fund for Cardiac Surgery.

\section{References}

1. Townsend N, Nichols M, Scarborough P, et al. Cardiovascular disease in Europe - epidemiological update 2015. Eur Heart $J$ 2015; 36: 2696-2705.
2. Vanhees L, Rauch B, Piepoli M, et al. writing group, EACPR. Importance of characteristics and modalities of physical activity and exercise in the management of cardiovascular health in individuals with cardiovascular disease (Part III). Eur J Prev Cardiol 2012; 19: 1333-1356.

3. Cornelissen VA, Onkelinx S, Goetschalckx K, et al. Exercise-based cardiac rehabilitation improves endothelial function assessed by flow-mediated dilation but not by pulse amplitude tonometry. Eur J Prev Cardiol 2014; 21: 39-48.

4. Oldridge N. Exercise-based rehabilitation in patients with coronary heart disease: Meta-analysis outcomes revisited. Future Cardiol 2012; 8: 729-751.

5. Mezzani A, Hamm LF, Jones AM, et al. European Association for Cardiovascular Prevention and Rehabilitation; American Association of Cardiovascular and Pulmonary Rehabilitation; Canadian Association of Cardiac Rehabilitation. Aerobic exercise intensity assessment and prescription in cardiac rehabilitation: A joint position statement of the European Association for Cardiovascular Prevention and Rehabilitation, the American Association of Cardiovascular Prevention and Rehabilitation and the Canadian Association of Cardiac Rehabilitation. Eur J Prev Cardiol 2013; 20: 442-467.

6. Rognmo Ø, Hetland E, Helgerud J, et al. High intensity aerobic interval exercise is superior to moderate intensity exercise for increasing aerobic capacity in patients with coronary artery disease. Eur J Cardiovasc Prev Rehabil 2004; 11: 216-222.

7. Warburton DE, McKenzie DC, Haykowsky MJ, et al. Effectiveness of high-intensity interval training for the rehabilitation of patients with coronary artery disease. Am J Cardiol 2005; 95: 1080-1084.

8. Rocco EA, Prado DM, Silva AG, et al. Effect of continuous and interval exercise training on the $\mathrm{PETCO}_{2}$ response during a graded exercise test in patients with coronary artery disease. Clinics 2012; 67: 623-627.

9. Currie KD, Dubberley JB, McKelvie RS, et al. Lowvolume, high-intensity interval training in patients with coronary artery disease. Med Sci Sports Exerc 2013; 45: 1436-1442.

10. Conraads VM, Pattyn N, De Maeyer C, et al. Aerobic interval training and moderate continuous training equally improve aerobic exercise capacity in patients with coronary artery disease: The SAINTEX-CAD study. Int J Cardiol 2015; 179: 203-210.

11. Moholdt TT, Amundsen BH, Rustad LA, et al. Aerobic interval training versus continuous moderate exercise after coronary artery bypass surgery: A randomized study of cardiovascular effects and quality of life. Am Heart $J$ 2009; 158: 1031-1037.

12. Keteyian SJ, Hibner BA, Bronsteen K, et al. Greater improvement in cardiorespiratory fitness using higherintensity interval training in the standard cardiac rehabilitation setting. J Cardiopulm Rehabil Prev 2014; 34: 98-105.

13. Cardozo CG, Oliveira RB and Farinatti PT. Effects of high intensity interval versus moderate continuous 
training on markers of ventilatory and cardiac efficiency in coronary heart disease patients. Scientific World Journal 2015; 192479.

14. Kim C, Choi HE and Lim MH. Effect of high interval training in acute myocardial infarction patients with drug-eluting stent. Am J Phys Med Rehabil 2015; 94: S879-S886.

15. Pattyn N, Vanhees L, Cornelissen VA, et al. The longterm effects of a randomized trial comparing aerobic interval versus continuous training in coronary artery disease patients: 1-Year data from the SAINTEX-CAD study. Eur J Prev Cardiol. 8 Feb 2016, ppi 2047487316631200.

16. Church TS, Earnest CP, Skinner JS, et al. Effects of different doses of physical activity on cardiorespiratory fitness among sedentary, overweight or obese postmenopausal women with elevated blood pressure: A randomized controlled trial. JAMA 2007; 297: 2081-2091.

17. Vanhees L, Stevens A, Schepers D, et al. Determinants of the effects of physical training and of the complications requiring resuscitation in patients with cardiovascular disease. Eur J Cardiovasc Prev Rehabil 2004; 11: 304-312.

18. Elliott AD, Rajopadhyaya K, Bentley DJ, et al. Interval training versus continuous exercise in patients with coronary artery disease: A meta-analysis. Heart Lung Circ 2015; 24: 149-157.

19. Pattyn N, Coeckelberghs E, Buys R, et al. Aerobic interval training vs. moderate continuous training in coronary artery disease patients: A systematic review and metaanalysis. Sports Med 2014; 44: 687-700.

20. Liou K, Ho S, Fildes J, et al. High intensity interval versus moderate intensity continuous training in patients with coronary artery disease: A meta-analysis of physiological and clinical parameters. Heart Lung Circ 2016; 25 : 166-174.

21. Dimopoulos S, Anastatiou-Nana M, Sakellariou D, et al. Effects of exercise rehabilitation program on heart rate recovery in patients with chronic heart failure. Eur $J$ Cardiovasc Prev Rehabil 2006; 13: 67-73.

22. Wisløff U, Støylen A, Loennechen JP, et al. Superior cardiovascular effect of aerobic interval training versus moderate continuous training in heart failure patients: A randomized study. Circulation 2007; 115: 3086-3094.

23. Iellamo F, Manzi V, Caminiti G, et al. Matched dose interval and continuous exercise training induce similar cardiorespiratory and metabolic adaptations in patients with heart failure. Int J Cardiol 2013; 167: 2561-2565.

24. Smart NA and Steele M. A comparison of 16 weeks of continuous vs intermittent exercise training in chronic heart failure patients. Congest Heart Fail 2012; 18: 205-211.

25. Conraads VM, Van Craenenbroeck EM, Pattyn N, et al. Rationale and design of a randomized trial on the effectiveness of aerobic interval training in patients with coronary artery disease: The SAINTEX-CAD study. Int $J$ Cardiol 2013; 168: 3532-3536.

26. Haddad M, Chaouachi A, Castagna C, et al. The convergent validity between two objective methods for quantifying training load in young taekwondo athletes. J Strength Cond Res 2012; 26: 206-209.

27. Williams JR. The declaration of Helsinki and public health. Bull World Health Organ 2008; 86: 650-652.

28. Mezzani A, Agostino P, Cohen-Solal A, et al. Standards for the use of cardiopulmonary exercise testing for the functional evaluation of cardiac patients: A report from the Exercise Physiology Section of the European Association for Cardiovascular Prevention and Rehabilitation. Eur J Cardiovasc Prev Rehabil 2009; 16: 249-267.

29. Wasserman K, Hansen JE, Sue DY, et al.. Normal values. In: Wasserman K, Hansen JE, Sue DY, et al. (eds) Principles of exercise testing and interpretation, 4th ed. Philadelphia: Lippincott Williams \& Wilkins, 2013, pp.160-182.

30. Binder RK, Wonisch M, Corra U, et al. Methodological approach to the first and second lactate threshold in incremental cardiopulmonary exercise testing. Eur $J$ Cardiovasc Prev Rehabil 2008; 15: 726-734.

31. Glass S and Dwyer GB. ACSM's metabolic calculations handbook., 7th ed. Philadelphia: Lippincott Williams \& Wilkins, 2007, p.145.

32. Moholdt T, Madssen E, Rognmo O, et al. The higher the better? Interval training intensity in coronary heart disease. J Sci Med Sport 2014; 17: 506-510.

33. Skinner JS and McLellan TH. The transition from aerobic to anaerobic metabolism. Res Q Exerc Sport 1980; 51: 234-248.

34. Perret $\mathrm{C}$ and Mueller G. Validation of a new portable ergospirometric device (Oxycon mobile) during exercise. Int J Sports Med 2006; 27: 363-367. 